\title{
Indian Society of Vascular and Interventional Radiology Expert Consensus Statements for Ablation in Hepatocellular Carcinoma: Part I
}

\author{
Pankaj Gupta1,@ Naveen Kalra ${ }^{1} \quad$ Shyamkumar N. Keshava² Sreedhara B. Chaluvashetty ${ }^{1}$ \\ Amar Mukund ${ }^{3}$ Shuvro H. Roy-Choudhury ${ }^{4}$ Sanjay Saran Baijal ${ }^{5} \quad$ Anubhav Khandelwal $^{5}$ \\ Venkatesh Hosur Ananthashayana ${ }^{3}$ Sathya Narayanan R. ${ }^{2}$ Suyash Kulkarni ${ }^{6}$ Nitin Sudhakar Shetty ${ }^{6}$ \\ Arun Gupta ${ }^{7}$ Sanjay Gupta ${ }^{8}$
}

\footnotetext{
${ }^{1}$ Department of Radiodiagnosis and Imaging, Postgraduate Institute of Medical Education and Research, Chandigarh, India

${ }^{2}$ Department of Interventional Radiology, Division of Clinical Radiology, Christian Medical College, Vellore, Tamil Nadu, India

${ }^{3}$ Department of Interventional Radiology, Institute of Liver and

Biliary Sciences, New Delhi, Delhi, India

${ }^{4}$ Department of Radiology, NH Group of Hospitals, Kolkata,

West Bengal, India

${ }^{5}$ Department of Interventional Radiology, Medanta, The Medicity, Gurgaon, Haryana, India

6 Tata Memorial Centre, Homi Bhabha National Institute, Mumbai, Maharashtra, India

${ }^{7}$ Department of Interventional Radiology, Sir Ganga Ram Hospital,

New Delhi, Delhi, India

${ }^{8}$ Department of Interventional Radiology, MD Anderson Cancer

Center, Houston, Texas, United States
}

\author{
Address for correspondence Shyamkumar N. Keshava, \\ DMRD, DNB, FRCR, FRANZCR, Department of Interventional \\ Radiology, Division of Clinical Radiology, Christian Medical College, \\ Vellore 632004, Tamil Nadu, India \\ (e-mail: aparna_shyam@yahoo.com).
}

J Clin Interv Radiol ISVIR:2020;4:98-106
Abstract
Keywords
- ablation
- hepatocellular carcinoma
- radiofrequency ablation

Locoregional therapies play an important role in the management of hepatocellular carcinoma (HCC). Percutaneous ablation is one of the most commonly employed nonsurgical methods for treating very early and early HCC. For small HCCs, ablation is potentially curative and competes with surgical resection. The widespread availability and the spectrum of ablative techniques mandate uniform approach among interventional radiologists. Thus, it is desirable to have a consensus regarding various aspects of liver ablation. This article represents a consensus document of the experts from the Indian Society of Vascular and Interventional Radiology involved in the care of patients with HCC. The statements are presented in two parts.

\section{Guiding Principle and Objectives}

Hepatocellular carcinoma (HCC) is the one of the most common cancers worldwide and is the leading cause of cancer-related mortality. ${ }^{1}$ The majority of HCCs occurs in the setting of cirrhosis and are diagnosed during surveillance. ${ }^{2}$ The therapies of HCC have evolved over the past 20 years. Broadly, the therapeutic options for HCC are hepatic resection (HR), liver transplantation, locoregional therapies including percutaneous ablation, transarterial chemoembolization (TACE), radioembolization, stereotactic body radiation therapy, and systematic therapies including immunotherapies. ${ }^{3}$ The treatment in an index patient is based on the tumor characteristics, including mainly the number and size of tumor nodules, the status of the portal veins, liver function, and performance status of the patient. Barcelona Clinic Liver Cancer (BCLC) represents one of the most widely used approaches when assessing a patient for management. ${ }^{3}$ Percutaneous ablation is a commonly utilized treatment for very early and early HCCs. ${ }^{4}$ There are several percutaneous ablation methods. The
DOI https://doi.org/ 10.1055/s-0040-1715774 ISSN 2457-0214.
License terms

()(1) $\Theta \circledast$ 
most commonly used is radiofrequency ablation (RFA), followed by microwave ablation (MWA), and cryoablation. The other techniques are irreversible electroporation, high intensity focused ultrasound, and laser ablation. As many tertiary care centers across India are offering liver tumor ablation, uniformity must be achieved. There is a lack of a document for the guidance of interventional radiologists involved in the ablation of HCCs.

The present consensus aimed to generate statements about various aspects of ablation procedures for HCCs to bridge a gap in the existing practice and the one supported by existing literature. The expert group comprising of interventional radiologists involved in the management of HCC performed multiple systematic reviews of the literature after selecting key questions. Grading of Recommendations Assessment, Development, and Evaluation (GRADE) system was used for grading evidence and strength of recommenda$\operatorname{tion}^{5}$ (-Table 1).

\section{Key Questions}

The guideline developers identified key questions that the interventional radiologists are faced with while considering patients with HCC for percutaneous ablation. Following questions were included:

1. What should be the aim of ablation?

2. Till what size, ablation may be performed?

3. What should be the target ablation margin?

4. Should adults with Child-Pugh C cirrhosis or performance status $>1$ undergo ablation?

5. What is the pre-procedure platelet count and international normalized ratio (INR) below which ablation is contraindicated?

6. Which imaging modality should be preferred for the guidance of ablation procedure?

7. Is there a role of fusion imaging or navigation techniques during an ablation?

8. What is the role of prophylactic antibiotics before ablation?

9. What strategies should be adopted for ablation of lesions at high risk locations?

10 . Should ascites be drained before ablation?

11. What is the role of transient vascular occlusion devices to prevent heat sink in lesions close to blood vessels?

12. Which is the preferred ablative method for perivascular tumors?

13. Should immediate postablation contrast imaging be routinely performed after ablation?

14. How should perihepatic hemorrhage postablation be managed?

15. What should be the protocol for postablation imaging?

16. What is the treatment of postablation residual or recurrent lesions?

17. What is the role of adjuvant therapies with ablation? This part of the article deals with the questions 1 to 10 .
Table 1 Grading of Recommendations Assessment, Development, and Evaluation (GRADE) approach

\begin{tabular}{|c|c|c|}
\hline $\begin{array}{l}\text { Rating the } \\
\text { quality of } \\
\text { evidence }\end{array}$ & Definition & $\begin{array}{l}\text { Type of } \\
\text { evidence }\end{array}$ \\
\hline High & $\begin{array}{l}\text { Further research is very } \\
\text { unlikely to change our confi- } \\
\text { dence in the estimate of effect }\end{array}$ & $\mathrm{RCT}$ \\
\hline Moderate & $\begin{array}{l}\text { Further research is likely to } \\
\text { have an important impact on } \\
\text { our confidence in the estimate } \\
\text { of effect and may change the } \\
\text { estimate }\end{array}$ & $\mathrm{RCT}$ \\
\hline Low & $\begin{array}{l}\text { Further research is very likely } \\
\text { to have an important impact } \\
\text { on our confidence in the } \\
\text { estimate of effect and is likely } \\
\text { to change the estimate }\end{array}$ & $\begin{array}{l}\text { Observational } \\
\text { study }\end{array}$ \\
\hline Very low & $\begin{array}{l}\text { Any estimate of effect is very } \\
\text { uncertain }\end{array}$ & $\begin{array}{l}\text { Any other } \\
\text { evidence }\end{array}$ \\
\hline $\begin{array}{l}\text { Rating the } \\
\text { strength of } \\
\text { recommen- } \\
\text { dations }\end{array}$ & Implications & Determinants \\
\hline Strong & $\begin{array}{l}\text { Population: Most people in } \\
\text { this situation would want } \\
\text { the recommended course } \\
\text { of action and only a small } \\
\text { proportion would not. } \\
\text { Health care workers: Most } \\
\text { people should receive the } \\
\text { recommended course of } \\
\text { action } \\
\text { Policy makers: The } \\
\text { recommendation can be } \\
\text { adapted as policy in most } \\
\text { situations }\end{array}$ & \multirow[t]{2}{*}{$\begin{array}{l}\text { Quality of } \\
\text { evidence } \\
\text { Balance of } \\
\text { benefit and } \\
\text { harm } \\
\text { Patient } \\
\text { values and } \\
\text { preferences } \\
\text { Resources and } \\
\text { costs }\end{array}$} \\
\hline Conditional & $\begin{array}{l}\text { Population: Most of the } \\
\text { people in this situation would } \\
\text { want the recommended } \\
\text { course of action, but many } \\
\text { would not } \\
\text { Health care workers: Be pre- } \\
\text { pared to help patients make } \\
\text { a decision that is consistent } \\
\text { with their values using deci- } \\
\text { sion aids and shared decision } \\
\text { making } \\
\text { Policy makers: There is a need } \\
\text { for substantial debate and } \\
\text { involvement of stakeholders }\end{array}$ & \\
\hline
\end{tabular}

Abbreviation: RCT, randomized controlled trial.

\section{What Should Be the Aim of Ablation?}

\section{Key Statements}

1. Ablation is performed with a potentially curative intent for very early (BCLC 0 ) and early stage (BCLC A) HCC patients. Quality/ certainty of Evidence: High

Strength of recommendation: Strong 


\section{Evidence and rationale}

Several studies have shown that RFA is safe and a locally curative procedure, which could be first-line treatment for selected patients with early HCC and potentially as a substitute for surgical resection of early stage HCC. A recent randomized controlled trial (RCT) comparing surgery ( $n=145)$ with RFA ( $n=148)$ for very early and early HCC showed that both surgery and RFA are equally effective (based on 3-year recurrence-free survival) and safe for HCCs $<3 \mathrm{~cm} .{ }^{6}$ After a median follow-up of 5 years, the recurrence-free survival was comparable in both groups (49.8\% for surgery and $47.7 \%$ for RFA; hazard ratio 0.96 , confidence interval (CI): $0.72-1.28 ; p=0.793$ ). The data for overall survival (OS) has not been published yet. One of the largest meta-analysis on this subject comprising 16,103 patients (31 studies with 3 RCTs and 28 observational studies), including 8,252 treated with RFA and 7,851 with HR for HCCs $\leq 3 \mathrm{~cm}$, reported that the disease-free survival (DFS) at 3 and 5 years, was significantly higher in HR group. ${ }^{7}$ The OS at 3 and 5 years for HR and RFA was $83.9 \%, 71.4 \%$ and $78.6 \%$ and $60.8 \%$, respectively. The odds ratio (OR) for OS was in favor of HR at both 3 years (OR: 0.65 , 95\% CI: $0.53-0.80$ ) and 5 years (OR: 0.57, 95\% CI: 0.48$0.67)$. The DFS at 3 and 5 years for HR and RFA was $56.7 \%$, $37.8 \%$ and $41.1 \%$, 26.6\%, respectively. However, for tumors $\leq 2 \mathrm{~cm}$, there was no significant difference in outcomes (OS at 5 years for HR and RFA was 74.2 and 69\%, respectively, and DFS at 5 years for HR and RFA was 41.6 and $42.5 \%$, respectively). The systematic review by Cucchetti et al did a critical analysis of 19 studies including 3 RCTs and 16 observational studies comparing HR with RFA. ${ }^{8}$ The authors concluded that potential biases and conflicting results of the available RCTs and observational studies demand well-designed studies as well as better statistical approach to balance the co-variates between the patient groups undergoing HR and RFA. A recent meta-analysis of 5 RCTs comparing HR and RFA for small HCCs showed no significant difference in OS at 1 year (relative risk, RR: $1.39,95 \% \mathrm{CI}: 0.36-5.33, p=0.53$ ) and 3 years (RR: 1.40 , 95\% CI: $0.75-2.62, p=0.29)$. However, at 5 years, RFA led to a significant decreased OS compared with HR (RR: 1.91, $95 \% \mathrm{Cl}: 1.32-2.79, p=0.0 .001)$. The incidence of intrahepatic recurrences as well as overall recurrences was significantly higher in RFA group (RR: $1.42,95 \%$ CI: 1.11-1.81, $p=0.005$ for intrahepatic recurrence and RR: $1.36 .95 \% \mathrm{CI}: 1.13-1.62$, $p=0.0009$ for overall recurrences). ${ }^{9}$ A network meta-analysis (74 studies, 20 RCTs, and 54 observational studies) comparing the treatment strategies for HCC showed that HR and RFA ranked one and two for OS at 1,3 , and 5 years for $\mathrm{HCC}<3 \mathrm{~cm}$ as well as those between 3 and $5 \mathrm{~cm}$. The pooled OR (95\% credible interval) of OS (RFA as compared with HR) from RCTs at 3 and 5 years for HCCs $<3 \mathrm{~cm}$ was $0.75(0.41-1.31)$ and $0.72(0.10-2.47)$, respectively. ${ }^{10}$ The pooled OR of OS from RCTs at 3 and 5 years for HCCs $3-5 \mathrm{~cm}$ was $0.24(0-1.25)$ and $1.05(0.03-5.33)$, respectively. There is one meta-analysis (1 RCT and 15 observational studies) comparing MWA and HR. ${ }^{11}$ In this study, MWA was associated with a significantly increased local tumor progression (LTP) rates compared with HR (RR: 2.49; 95\% CI: 1.19-5.22, $p=0.016$ ). Additionally, the 3- and 5-year OS (RR: 0.94, $p=0.03$ and RR: 0.88, $p=0.01$ ) and 3 years DFS (RR:0.78; $p=0.009$ ) were significantly higher for HR. No significant difference was reported for OS $(\mathrm{RR}=1.01, p=0.409)$ and DFS (RR: 0.95, $p=0.085)$ at 1 year. Moreover, the DFS was not significantly different (RR: 0.83 , $p=0.284$ ) between the two groups at 5 years.

\section{Till What Size, Ablation May Be Performed?}

\section{Key Statements}

- Ablation may be considered as a first-line treatment for very early HCCs up to $2 \mathrm{~cm}$ (BCLC 0 ) who are not candidates for liver transplantation.

- Ablation can be considered as an acceptable alternative to surgery or transplantation for HCC up to $3 \mathrm{~cm}$ (BCLC A), in surgically unfit cases or according to patient choice.

- Ablation in combination with TACE may also be offered for larger lesions (between 3 and $5 \mathrm{~cm}$ ) (BCLC A) when resection or transplant is contraindicated.

\section{Quality/certainty of Evidence: High}

Strength of recommendation: Strong

\section{Evidence and rationale:}

A systematic review and meta-analysis including 17 studies (3,996 patients treated with resection and 4,424 patients treated with ablation) concluded that for very early HCC (single nodule $<2 \mathrm{~cm}$ ) in Child-Pugh class A patients, RFA provided similar 3 years OS (RR: 0.99, 95\% CI: 0.83-1.19, $p=0.949)$ and DFS (RR: $1.18,95 \%$ CI: $0.88-1.58, p=0.263$ ) at a lower cost. ${ }^{12}$ Recent data of long-term survival supports the effectiveness of RFA for small tumors. In the study by Lee et al, 5- and 10-year OS of patients with single $\mathrm{HCC}<3 \mathrm{~cm}$ treated with RFA was reported to be 83.7 and $74.2 \%$, respectively. ${ }^{13} \mathrm{At}$ 5 - and 10 years, LTP rates were 20.5 and $25.3 \%$. The studies discussed in the section above also support the role of RFA as first-line treatment for very early $\mathrm{HCC} \leq 2 \mathrm{~cm} .^{7-11}$

For BCLC A lesions (lesions within Milan criteria), ablation can be considered as an alternative to surgery or transplantation, in surgically unfit cases or according to patient choice, based on the multidisciplinary local experience. In the RCT by Huang et al, 230 patients were randomly assigned to receive RFA and HR. Patients were followed for 5 years. HR was found to be associated with significantly better OS ( $p=$ $0.001)$ and DFS $(p=0.017) .{ }^{14}$ Recurrences were reported in 73 patients in the RFA and 48 patients in the HR group $(p=$ 0.001 ). The 1-, 2-, 3-, 4-, and 5-year overall recurrence rates were $16.52,38.26,49.57,59.13$, and $63.48 \%$ for the RFA group and $12.17,22.60,33.91,39.13$, and $41.74 \%$ for the HR group, respectively. No subgroup analysis was done based on the size of tumor; however, on univariate and multivariate analysis, the tumor size $(\leq 3 \mathrm{~cm}$ or $>3 \mathrm{~cm})$ was not significantly associated with recurrence. However, in the recent RCTs by Fang et al and $\mathrm{Ng}$ et al, there was no significant difference in the LTP, OS, and DFS between the patient groups undergoing RFA and HR. ${ }^{15,16}$ Fang et al, randomly assigned 120 patients to RFA or HR. At a mean follow-up of 40 months, the local recurrence rate was $36.6 \%$ in the RFA arm and $35 \%$ in the HR $\operatorname{arm}(p>0.05) .{ }^{15}$ The OS and DFS at 1,2 , and 3 years were not 
significantly different ( $p=0.207$ and $p=0.443$, respectively). In the study by $\mathrm{Ng}$ et al comprising 109 patients with early HCC (within Milan criteria) in each arm, there was no significant difference in the $1,3,5$, and 10 years OS $(p=0.531){ }^{16}$ Similarly, the DFS rates were also comparable at $1,3,5$, and 10 years $(p=0.072)$. There was no difference in the pattern of recurrence, early ( $\leq 2$ years), or late recurrence ( $>2$ years) between the two groups ( $p=0.092, p=0.334, p=0.568$, respectively). The early recurrence rate was $37 \%$ in HR group versus $43.1 \%$ in the RFA group. The late recurrence rate was $34.3 \%$ in HR group versus $38.5 \%$ in the RFA group. At multivariate analysis, tumor size $>3 \mathrm{~cm}$ was associated with poor DFS. In a recent network meta-analysis (of 36 RCTs) comparing the various treatment strategies for HCC within Milan criteria, total recurrence was reported to be more common with RFA compared with HR at 1 year (RR: 0.67, 95\% CI: 0.27-1.6), 2 years (RR: $0.64,95 \% \mathrm{CI}: 0.30-1.4$ ), 3 years (RR: $0.76,95 \% \mathrm{Cl}$ : $0.5-1.1$ ), and $>3$ years (RR: 0.81, 95\% CI: $0.54-1.2$ ). However, no subgroup analysis based on the size of HCC was done. ${ }^{17}$

Additionally, all the RCTs have reported that RFA has lesser treatment duration, lesser blood loss, and shorter hospital stay. ${ }^{14-16}$

Some patients with larger HCCs may not be suitable to undergo HR or transplant. Ablation in combination with TACE may allow effective management of these patients. In the prospective study by Saviano et al solitary large HCCs (median size $4 \mathrm{~cm}$ and range, $3-7.4 \mathrm{~cm}$ ) were treated with RFA plus TACE. ${ }^{18}$ There was no difference in the OS at 1 - and 3 years. Although, the tumor recurrence and LTP was higher in the RFA plus TACE group compared with the HR group, the former group had patients with more severe portal hypertension. Another study reported the effectiveness of RFA plus drug eluting bead TACE for large HCCs not amenable to surgical resection. ${ }^{19}$ There is an ongoing RCT comparing OS and DFS of HR with TACE plus RFA for BCLC Stage B HCC. ${ }^{20}$

\section{What Should Be the Target Ablation Margin?}

\section{Key Statement}

The target ablative margin should be at least $10 \mathrm{~mm}$ circumferentially around the entire lesion.

Quality/ certainty of Evidence: Moderate

Strength of recommendation: Strong

\section{Evidence and rationale}

The term "ablative margin" refers to the rim of parenchyma that is ablated beyond the tumor margin with the aim to achieve complete tumor necrosis. Liao et al conducted an RCT to compare the outcomes following a wide margin (ablative margin $\geq 10 \mathrm{~mm}$ ) and narrow margin (ablative margin, $5-10 \mathrm{~mm}$ ). ${ }^{21}$ Patients with solitary HCC less than $3 \mathrm{~cm}$ were randomized to receive a wide margin $(n=48)$ or narrow margin ablation $(n=48)$. Forty patients in the former group achieved the target of $\geq 10 \mathrm{~mm}$ ablative margin. The ablative margin was monitored by 3D registration on computed tomography (CT). The LTP and intrahepatic recurrence at 3 years were significantly lower and recurrence-free survival significantly higher for wide margin ablation. Based on these results, the authors concluded that RFA treatment targeting a $10-\mathrm{mm}$ ablative margin might decrease the risk of local tumor recurrence in patients with small HCC. In another study by Fukuda et al comprising 85 HCC nodules in 76 patients, superparamagnetic iron oxide magnetic resonance imaging (MRI) was performed pre- and postprocedure (within 4-7 days). ${ }^{22}$ At 3 years, LTP was $2 \%$ when an ablative margin had been achieved around the lesion compared with $34 \%$ when no ablative margin has been achieved. When there was an ablation margin $\geq 2 \mathrm{~mm}$, no recurrences were reported. In a study by Kim et al, an ablative margin of $5 \mathrm{~mm}$ was attempted during RFA of 110 HCC nodules in 103 patients. ${ }^{23}$ The ablative margin was assessed using fused CT images. At 1-year follow-up, LTP was reported to be 22.7, $18.9,5.9$, and $0 \%$ when the ablative margin was $\geq 0 \mathrm{~mm}$, $\geq 1 \mathrm{~mm}, \geq 2 \mathrm{~mm}$, and $\geq 3 \mathrm{~mm}$. Based on this, the authors concluded that ablative margin $\geq 3 \mathrm{~mm}$ is associated with lower rates of local tumor recurrence.

\section{Should Adults with Child-Pugh C Cirrhosis or Performance Status $>1$ Undergo Ablation?}

\section{Key Statements}

1. Ablation can be offered to selected patients with ChildPugh C cirrhosis and HCC.

2. Molecular targeted therapy or chemotherapy is recommended as a standard treatment for HCC patients with performance status (PS) 1 to 2 according to the BCLC system. However, ablation can be offered to selected patients with PS 1 to 2, if there are no contraindications.

Quality/certainty of evidence: Low

Strength of recommendation: Conditional

\section{Evidence and rationale}

In a study comparing the outcomes in patients with ChildPugh C HCC who underwent locoregional therapies versus those who underwent the best supportive care (BSC), a survival advantage was recorded in the former group. ${ }^{24}$ The median survival time was 0.95 years (treatment group) versus 0.17 years (BSC). In another study, the safety and efficacy of RFA in the treatment of HCC in the setting of decompensated cirrhosis (Child-Pugh score $>9$ ) were evaluated. ${ }^{25}$ Twenty-six HCCs in 19 patients were treated with RFA. The mean ChildPugh score was 10.7 , and the mean tumor diameter was $1.96 \mathrm{~cm}$. The median OS was $12.0 \pm 1.7$ months. Complete necrosis without any marginal recurrence was reported in $88.5 \%$ of patients. Two patients died of liver failure, 2 and 4 months following therapy. In a study by Nouso et al, prognostic factors in $157 \mathrm{HCC}$ patients with Child-Pugh C cirrhosis were evaluated. High serum bilirubin ( $>3 \mathrm{mg} / \mathrm{dL}$ ), refractory ascites, platelet count $\left(>8 \times 10^{4} / \mathrm{mm}^{3}\right)$, multiple tumors, large tumors $(>3 \mathrm{~cm})$, high $\alpha$-fetoprotein $(>400 \mathrm{ng} / \mathrm{mL}$ ), and portal vein thrombus were associated with poor prognosis. ${ }^{26}$ Wakuta et al reported the safety and efficacy of RFA for HCC in patients with Child-Pugh B $(n=53) / C(n=10)$ cirrhosis. ${ }^{27}$ The OS in Child-Pugh B and C cirrhosis was 82 and $83 \%$ at 1 year and 47 and $31 \%$ at 3 years, respectively. At 1,3 , 
and 6 months after RFA, there were no significant changes in components of Child-Pugh score (albumin, total serum bilirubin, prothrombin time, ascites, and encephalopathy) in patients with Child-Pugh B cirrhosis. There was a significant increase in serum bilirubin levels at 6 months after RFA in $60 \%$ of patients with Child-Pugh C cirrhosis. In another study, the effect of RFA on liver parenchymal function was reported. Liver function tests and complications were monitored until 6 months after treatments. ${ }^{28}$ Patients with a high Child-Pugh score $(\geq 8)$ before treatment showed elevation of Child-Pugh score more frequently when compared with those who had a low pre-treatment score $(<8)$. Refractory ascites was reported only in patients with a high pretreatment Child-Pugh score. The authors suggested that patients with a pre-treatment Child-Pugh score $\geq 8$ are not good candidates for RFA. A study by Kuroda et al supported these findings and indicated that Child-Pugh score $\geq 9$ is a significant risk factor for long-term deterioration of liver function following RFA. ${ }^{29}$

In a study by Hsu et al comparing the aggressive therapy (ablation, TACE, HR, and transplantation) with the BSC using a propensity score matching, 507 patients received aggressive anticancer treatment, and 286 patients received BSC. ${ }^{30}$ Ablation was performed in 117 (22\%) patients. It was found that patients who received aggressive treatment had significantly better long-term survival (hazard ratio 2.028, $p<0.0001$ ). In another study by the same group, patients with PS2 were divided into two matched groups: those receiving aggressive anticancer treatment and BSC. ${ }^{31}$ Although patients received aggressive treatment had better long-term survival, no survival benefit was found after adjusting for the confounding factors in the propensity-matched model. However, in the published studies, the effect of PS on the efficacy and complications of ablation per se has not been reported.

\section{What Is the Pre-Procedure Platelet Count and INR below Which Ablation Is Contraindicated?}

\section{Key Statement}

Correction of the respective parameters prior to ablation is required for platelet count less than $50000 / \mu \mathrm{L}$ and INR $>1.5$

Quality/ certainty of evidence: Low

Strength of recommendation: Conditional

\section{Evidence and rationale}

The evidence regarding the use of pre-procedure testing of coagulogram prior to ablation is lacking. There are no RCTs controlled specific to ablation procedures. Society of Interventional Radiology (SIR) guidelines place the percutaneous ablation procedures into either moderate or severe risk of bleeding depending on the complexity of ablation. ${ }^{32}$ For straightforward ablation procedures, routine pre-procedure testing of platelet count is not recommended. However, testing of INR should be routinely done. In complex ablations posing a severe risk of bleeding, pre-procedure testing of both platelet count and INR is recommended. The SIR recommendations, however, are based on Delphi consensus by a panel of experts. Moreover, the definition of "complex" ablations is not given. In a study by Goto et al evaluating the hemorrhagic complications of RFA, large tumor size and low platelet count were the risk factors for hemoperitoneum. ${ }^{33}$ Lee et al reported thrombocytopenia (platelet count $<100,000 / \mathrm{mm}^{3}$ ) to be a significant risk factor for deterioration of liver function following RFA. ${ }^{34}$ In a meta-analysis by Pang et al, low level of platelet count before the ablation had significant association with poor survival as shown by the pooled hazard ratios for OS and recurrence-free survival of 1.41 (95\% CI: $1.14-1.75)$ and 1.44 (95\% CI: 1.13-1.83), respectively. ${ }^{35}$ INR should be corrected to $<1.5$ by the transfusion of fresh-frozen plasma (FFP) and platelet count should be corrected to $>50000 / \mu L^{32}$ However, recent studies have questioned this strategy. According to the recent American clinical practice update, administration of large volume of FFP should not be used to reach threshold values of INR. ${ }^{36}$ This is due to the limitations of usual targets proposed for INR, minimal effect of FFP on thrombin as well as potential for worsening of portal hypertension. The use of thromboelastography (TEG), rather than the INR and platelet count, has been proposed to be a better indicator of the need for FFP or platelet transfusion prior to invasive procedures. A RCT showed that use of TEG-guided transfusion strategy led to a significantly reduced need for blood products. ${ }^{37}$

\section{Which Imaging Modality Should Be Preferred for Guidance of Ablation Procedure?}

\section{Key Statement}

The tumor ablation may be performed under US, CT, or MR guidance based on tumor visibility, local availability of dedicated equipment, and operator preference as well as experience.

Quality/certainty of evidence: Low

Strength of recommendation: Conditional

\section{Evidence and rationale}

In a retrospective study by Lee et al, RFA was performed in 152 patients: 101 patients underwent RFA under US guidance, and 51 patients underwent RFA under CT guidance. All the patients had complete tumor ablation..$^{38} \mathrm{~A}$ median of two sessions was required for the US group compared with one session for the CT group $(p<0.001)$. One, two, and three years tumor recurrence and OS rates were not significantly different between the two groups. Both groups had similar complication rates. In a study by Kitada et al, 63 patients with solitary HCC were included. RFA under conventional US guidance and real-time virtual sonography guidance were performed in 39 and 24 patients, respectively. ${ }^{39}$ The technical success and local recurrence rate were similar in both groups. In a study by Kim et al, 109 patients with 136 HCC were evaluated with the US. ${ }^{40}$ Forty-five patients were deemed nonfeasible for treatment under US guidance due to tumor nonvisibility ( $n=32$ ), inability to identify safe path $(n=5)$, high risk of damage to adjacent organs $(n=5)$, or a combination of these factors $(n=3)$. The inability to visualize the lesions was related to the small size and inability to differentiate tumor nodules from the background macronodular cirrhosis. 
MR-guided ablation has been described to be safe and effective with a need for fewer sessions. In 2007, Clasen et al demonstrated the feasibility and technical effectiveness of MR-guided RFA. ${ }^{41}$ In this study, 64 patients with 100 tumors (19 HCCs and 81 metastases) were treated with 87 sessions on a 0.2-T open MRI. Technical success was achieved in $97.7 \%$ cases, and complete ablation was achieved in $99 \%$ cases. There were $2.3 \%$ major and $8 \%$ minor complications. In another study by Clasen et al, 20 patients with $28 \mathrm{HCC}$ nodules underwent 25 sessions of MR-guided RFA on a $0.2 \mathrm{~T}$ open MR system. ${ }^{42}$ Technical success was achieved in $100 \%$ cases, and complete ablation rate was $96.4 \%$. There was one major and one minor complication. In yet another study, Clasen et al compared 53 CT-guided RFA procedures with 24 MR-guided procedures. ${ }^{43}$ Technical effectiveness was achieved in 79.3\% CT-guided and 96.3\% MR RFA procedures $(p=0.04)$. The authors concluded that MR-guided RFA may require fewer sessions for complete tumor ablation. In a recent study, Yuan et al compared US, CT, and MR-guided RFA and found no difference in the technical success, technical effectiveness as well as PFS or OS. ${ }^{44}$

\section{Is There a Role of Fusion Imaging or Navigation Techniques during Ablation?}

\section{Key Statement}

Image-fusion and navigation systems may be used for tumor targeting in a difficult location.

Quality/certainty of evidence: Low

Strength of recommendation: Conditional

\section{Evidence and rationale}

Fusion imaging and navigation techniques have been described for precise targeting of the lesions in difficult locations. In a study by Lee et al, 30 patients with 30 HCC nodules were evaluated with fusion imaging. ${ }^{45}$ Twenty-seven (90\%) lesions could be targeted for RFA with fusion imaging. The authors concluded that fusion image-guided RFA is effective in lesions that have poor conspicuity on US. Song et al evaluated the US/MR fusion-guided RFA of recurrent subcentimetric sized HCC nodules. ${ }^{46}$ In this study comprising 181 patients, fusion image-guided RFA was feasible in $65.7 \%$ of the patients. The technical success in patients who underwent RFA was $98.4 \%$. In a study of 20 patients by Krücker et al, electromagnetic needle tracking was investigated for percutaneous US-guided RFA $(n=8) /$ biopsy $(n=12) .{ }^{47}$ There was a mean tracking error of $4.7 \pm 1.5 \mathrm{~mm}$ in patients who underwent liver RFA/ biopsy. The authors concluded that the accuracy of spatial tracking is sufficient for the preprocedural display of imaging information for guiding procedures. In another study, the feasibility of real-time virtual needle tracking during percutaneous RFA was assessed. In eight patients with eight HCC nodules, the needle tip could be accurately localized with virtual needle tracking. ${ }^{48}$

In another study, stereotactic CT-guided MWA of the liver tumor using high-frequency jet ventilation was reported. ${ }^{49}$ Twenty patients with 28 HCCs that were not visible or accessible on US were included. The mean lateral tracking error was $14.9 \pm 5.9 \mathrm{~mm}$, depth error was $3.4 \pm$ $3.2 \mathrm{~mm}$, and the total error was $5.8 \pm 3.2 \mathrm{~mm}$. There were no major complications and no requirement for needle repositioning.

\section{What Is the Role of Prophylactic Antibiotics before Ablation?}

\section{Key Statement}

The routine use of prophylactic antibiotics for liver ablation is not recommended. The antibiotic prophylaxis should be considered for patients at high risk of infection including those with biliary abnormalities (e.g., bilioenteric anastomosis), large centrally located tumors near central bile ducts, and uncontrolled diabetes mellitus.

Quality/certainty of evidence: Low

Strength of recommendation: Conditional

\section{Evidence and rationale}

In the study by Odisio et al, 262 patients underwent 307 sessions of percutaneous ablation of liver tumors. ${ }^{50}$ Twelve patients had a prior hepaticojejunostomy. Ten patients received aggressive prophylactic antibiotic regimen. Two received other antibiotic regimens. None of the patients receiving aggressive prophylactic antibiotic regimen developed liver abscesses. However, both patients receiving the other antibiotic regimen developed abscesses. In another study by Bhatia et al, 123 RFA procedures were performed in 83 patients..$^{51}$ None of the patients had the risk factors like prior bilioenteric anastomoses, biliary tract abnormalities, or uncontrolled diabetes mellitus. No prophylactic antibiotics were given for 121 sessions. Only one patient in this group developed an abscess.

\section{What Is the Role of "Artificial" Ascites during Ablation?}

\section{Key Statement}

Artificial ascites is safe and effective technique to improve lesion visualization and minimize injury to adjacent structures when ablating HCCs at high-risk locations.

Quality/certainty of evidence: Low

Strength of recommendation: Conditional

\section{Evidence and rationale}

Thermal ablative techniques for treating HCC expose the areas surrounding the lesion to conductive heat and may result in collateral thermal damage. This is of concern when the lesion is abutting the structures, which are extrahepatic such as gallbladder, gastrointestinal (GI) tract, pericardium, or diaphragm. Livraghi et al reported that perforation of the GI tract is one of the most important major complications due to thermal damage. ${ }^{52}$ This was more likely with a history of prior GI surgery. Thus, it is important to use ablative treatment with due precaution to safeguard these structures from collateral damage by achieving thermal insulation between the hepatic lesion being ablated and 
the abutting extrahepatic structures. One way to achieve this is by having fluid between the structures that work as an insulator and protect the adjacent structures from transmitted heat. Efficacy and safety of artificial ascites and artificial pleural effusion in protecting the adjacent structures have been demonstrated in earlier studies. ${ }^{53-57}$ Hsieh et al showed that higher technical effectiveness, fewer complications, and LTP with improved OS may be achieved with creation of artificial ascites and/or pleural effusion for HCCs in high-risk locations. ${ }^{53}$ In this study, 138 patients with 195 tumors were evaluated. Artificial ascites or artificial pleural effusion was created in 48 patients with 76 tumors. ${ }^{53}$ The mean volume of intraperitoneal or intrapleural fluid used was $822 \mathrm{~mL}$ (range, $350-1750 \mathrm{~mL}$ ). The primary technical effectiveness was higher in the group that underwent the creation of artificial ascites or pleural effusion (89.5 vs. $76.5 \%, p=0.046)$. Fewer complications were recorded in this group. LTP rates at 12 and 24 months were significantly lower, and OS significantly higher in this group. In another study by Zhang et al, safety of MWA in HCC adjacent to the GI tract treated after creating artificial ascites was demonstrated. ${ }^{54}$ In this study, artificial ascites was created in 36 out of 761 patients with HCCs located $<5 \mathrm{~mm}$ from the GI tract. A volume of 100 to $1500 \mathrm{~mL}$ normal saline was injected to achieve at least $5 \mathrm{~mm}$ separation between the tumor and the adjacent GI tract. The separation success rate was $88.9 \%$ (32/36). The ablation was technically effective in $96.9 \%$ of cases. During a mean follow-up of $12.1 \pm 7.2$ months, LTP was observed in five patients. No major complications related to artificial ascites were encountered. Similar safety was reported for RFA of HCCs adjacent to the GI tract with the creation of artificial ascites. ${ }^{55}$ Song et al evaluated the utility of artificial ascites in RFA of lesions abutting the diaphragm and GI tract. ${ }^{55}$ In this study comprising 143 patients with 181 HCCs, 148 lesions were found to be in high-risk locations related to poor sonological window or potential for thermal injury. In these cases, artificial ascites was created using $5 \%$ dextrose. Complete necrosis was achieved in $91.2 \%$ of cases (35/148 nodules). Hermida et al assessed the safety of carbon dioxide pneumothorax for the ablation of HCCs abutting the domes of the diaphragm..$^{56}$ In this study comprising 56 HCCs, 28 HCC underwent US-guided ablation without $\mathrm{CO}_{2}$ instillation, while $28 \mathrm{HCCs}$ were ablated under $\mathrm{CT}$ guidance after $\mathrm{CO}_{2}$ instillation. Primary technical success was $100 \%$ in both groups. No major complications occurred. After a median follow-up of 13.8 months, LTP was recorded in a smaller number of patients in the $\mathrm{CO}_{2}$ group (10.7 vs. $25 \%$ ). Artificial ascites has been reported to be safe. Nishimura et al reported that there was no significant difference in the rate of complications with increasing volumes of artificial ascites. ${ }^{57}$ They divided patients into three groups: no artificial ascites $(n=86),<1 \mathrm{~L}$ of ascites $(n=35)$, and $>1 \mathrm{~L}$ of ascites $(n=67)$. No severe complications such as lung injury, pneumothorax, intra-peritoneal bleeding, GI perforation, and biliary injury were reported in any group. Minor complications were reported only in group I. There was no significantdifferenceintherateoflocal recurrencebetweenthe three groups.

\section{Should Ascites Be Managed before Ablation?}

\section{Key Statement}

Ascites need not be drained before ablation since performing RFA is safe, even when a large volume of artificial ascites is created. However, the presence of ascites may signify poor liver function, and the decision to perform RFA in such cases should consider the Child-Pugh score.

Quality/certainty of evidence: Low

Strength of recommendation: Conditional

\section{Evidence and rationale}

The risk of bleeding is one of the most common and dreaded complications from any percutaneous intervention in the liver, and the same holds for percutaneous ablation for liver tumors, as reported in earlier studies. ${ }^{52}$ A percutaneous intervention like liver biopsy in the presence of ascites has been considered a relative contraindication due to increased risk of bleeding. Percutaneous ablation of liver tumors in the presence of ascites may raise similar concerns for the possibility of bleeding from the puncture site on the liver surface. However, various studies have reported the safety of RFA for liver tumors in cases of ascites, where precise tract ablation is performed ${ }^{58,59}$ Doppler US in the presence of ascites can easily and precisely demonstrate the site of bleeding from the liver surface, and additional ablation of this site can then be performed to cauterize the bleeding site. In a study by Cha et al, 19 patients with ascites underwent RFA of HCC. ${ }^{58}$ This study comprised six subcapsular lesions. No major complications were recorded. Hemoperitoneum occurred in two patients, both with subcapsular lesions. However, there was no extension in the duration of hospital stay. No postprocedure hemorrhage was recorded in 23 sessions of MWA in 19 patients in another study by Sherwani et al. ${ }^{59}$

\section{Financial Disclosures \\ None. \\ Conflict of Interest \\ None.}

\section{References}

1 White DL, Thrift AP, Kanwal F, Davila J, El-Serag HB. Incidence of hepatocellular carcinoma in all 50 United States, from 2000 through 2012. Gastroenterology 2017;152(4):812-820.e5

2 Bruix J, Reig M, Sherman M. Evidence-based diagnosis, staging, and treatment of patients with hepatocellular carcinoma. Gastroenterology 2016;150(4):835-853

3 Heimbach JK, Kulik LM, Finn RS, et al. AASLD guidelines for the treatment of hepatocellular carcinoma. Hepatology 2018;67(1):358-380

4 Shiina S, Sato K, Tateishi $\mathrm{R}$, et al. Percutaneous ablation for hepatocellular carcinoma: comparison of various 
ablation techniques and surgery. Can J Gastroenterol Hepatol 2018;2018:4756147

5 Atkins D, Best D, Briss PA, et al; GRADE Working Group. Grading quality of evidence and strength of recommendations. BMJ 2004;328(7454):1490

6 Izumi N, Hasegawa K, Nishioka Y, et al. A multicenter randomized controlled trial to evaluate the efficacy of surgery vs. radiofrequency ablation for small hepatocellular carcinoma (SURF trial) J Clin Oncol 2019;37(15):4002

7 Xu Q Kobayashi S, Ye X, Meng X. Comparison of hepatic resection and radiofrequency ablation for small hepatocellular carcinoma: a meta-analysis of 16,103 patients. Sci Rep 2014;4:7252

8 Cucchetti A, Piscaglia F, Cescon M, Ercolani G, Pinna AD. Systematic review of surgical resection vs radiofrequency ablation for hepatocellular carcinoma. World J Gastroenterol 2013;19(26):4106-4118

9 Xu XL, Liu XD, Liang M, Luo BM. Radiofrequency ablation versus hepatic resection for small hepatocellular carcinoma: systematic review of randomized controlled trials with meta-analysis and trial sequential analysis. Radiology 2018;287(2):461-472

10 Tian G, Yang S, Yuan J, et al. Comparative efficacy of treatment strategies for hepatocellular carcinoma: systematic review and network meta-analysis. BMJ Open 2018;8(10):e021269

11 Glassberg MB, Ghosh S, Clymer JW, Wright GWJ, Ferko N, Amaral JF. Microwave ablation compared with hepatic resection for the treatment of hepatocellular carcinoma and liver metastases: a systematic review and meta-analysis. World J Surg Oncol 2019;17(1):98

12 Cucchetti A, Piscaglia F, Cescon M, et al. Cost-effectiveness of hepatic resection versus percutaneous radiofrequency ablation for early hepatocellular carcinoma. J Hepatol 2013;59(2):300-307

13 Lee MW, Kang D, Lim HK, et al. Updated 10-year outcomes of percutaneous radiofrequency ablation as first-line therapy for single hepatocellular carcinoma $<3 \mathrm{~cm}$ : emphasis on association of local tumor progression and overall survival. Eur Radiol 2020;30(4):2391-2400

14 Huang J, Yan L, Cheng Z, et al. A randomized trial comparing radiofrequency ablation and surgical resection for HCC conforming to the Milan criteria. Ann Surg 2010;252(6):903-912

15 Fang Y, Chen W, Liang X, et al. Comparison of long-term effectiveness and complications of radiofrequency ablation with hepatectomy for small hepatocellular carcinoma. J Gastroenterol Hepatol 2014;29(1):193-200

$16 \mathrm{Ng} \mathrm{KK}$, Chok KSH, Chan ACY, et al. Randomized clinical trial of hepatic resection versus radiofrequency ablation for early-stage hepatocellular carcinoma. Br J Surg 2017;104(13):1775-1784

17 Lin Y, Wen Q, Guo L, Wang H, Sui G, Sun Z. A network meta-analysis on the efficacy and prognosis of different interventional therapies for early-stage hepatocellular carcinoma. Int J Hyperthermia 2018;35(1):450-462

18 Saviano A, Iezzi R, Giuliante F, et al; HepatoCATT Study Group. Liver resection versus radiofrequency ablation plus transcatheter arterial chemoembolization in cirrhotic patients with solitary large hepatocellular carcinoma. J Vasc Interv Radiol 2017;28(11):1512-1519

19 Iezzi R, Pompili M, La Torre MF, et al; HepatoCATT Study Group for the Multidisciplinary Management of HCC. Radiofrequency ablation plus drug-eluting beads transcatheter arterial chemoembolization for the treatment of single large hepatocellular carcinoma. Dig Liver Dis 2015;47(3):242-248

20 Peng B. Hepatic Resection Versus Transarterial Chemoembolization Plus Radiofrequency Ablation for Barcelona Clinic Liver Cancer Stage B Hepatocellular
Carcinoma (NCT02616926). Available at: https://clinicaltrials. gov/ct2/show/NCT02616926. Accessed August 5, 2020

21 Liao M, Zhong X, Zhang J, et al. Radiofrequency ablation using a $10-\mathrm{mm}$ target margin for small hepatocellular carcinoma in patients with liver cirrhosis: a prospective randomized trial. J Surg Oncol 2017;115(8):971-979

22 Fukuda K, Mori K, Hasegawa N, et al. Safety margin of radiofrequency ablation for hepatocellular carcinoma: a prospective study using magnetic resonance imaging with superparamagnetic iron oxide. Jpn J Radiol 2019;37(7):555-563

23 Kim YS, Lee WJ, Rhim H, Lim HK, Choi D, Lee JY. The minimal ablative margin of radiofrequency ablation of hepatocellular carcinoma ( $>2$ and $<5 \mathrm{~cm}$ ) needed to prevent local tumor progression: 3D quantitative assessment using $\mathrm{CT}$ image fusion. AJR Am J Roentgenol 2010;195(3):758-765

24 Kim YK, Kim CS, Chung GH, et al. Radiofrequency ablation of hepatocellular carcinoma in patients with decompensated cirrhosis: evaluation of therapeutic efficacy and safety. AJR Am J Roentgenol 2006;186(5, Suppl):S261-S268

25 Nishikawa H, Kita R, Kimura T, et al. Clinical efficacy of non-transplant therapies in patients with hepatocellular carcinoma with Child-Pugh C liver cirrhosis. Anticancer Res 2014;34(6):3039-3044

26 Nouso K, Ito Y, Kuwaki K, et al. Prognostic factors and treatment effects for hepatocellular carcinoma in Child C cirrhosis. Br J Cancer 2008;98(7):1161-1165

27 Wakuta A, Nouso K, Kariyama K, et al. Radiofrequency ablation for the treatment of hepatocellular carcinoma with decompensated cirrhosis. Oncology 2011;81(1):39-44

28 Koda M, Ueki M, Maeda Y, et al. The influence on liver parenchymal function and complications of radiofrequency ablation or the combination with transcatheter arterial embolization for hepatocellular carcinoma. Hepatol Res 2004;29(1): $18-23$

29 Kuroda H, Kasai K, Kakisaka K, et al. Changes in liver function parameters after percutaneous radiofrequency ablation therapy in patients with hepatocellular carcinoma. Hepatol Res 2010;40(5):550-554

30 Hsu CY, Liu PH, Lee YH, et al. Aggressive therapeutic strategies improve the survival of hepatocellular carcinoma patients with performance status 1 or 2: a propensity score analysis. Ann Surg Oncol 2015;22(4):1324-1331

31 Hsu CY, Liu PH, Lee YH, et al. Hepatocellular carcinoma patients with performance status 1 deserve new classification and treatment algorithm in the BCLC system. Medicine (Baltimore) 2015;94(29):e1223

32 Patel IJ, Davidson JC, Nikolic B, et al; Standards of Practice Committee, with Cardiovascular and Interventional Radiological Society of Europe (CIRSE) Endorsement. Consensus guidelines for periprocedural management of coagulation status and hemostasis risk in percutaneous image-guided interventions. J Vasc Interv Radiol 2012;23(6): 727-736

33 Goto E, Tateishi R, Shiina S, et al. Hemorrhagic complications of percutaneous radiofrequency ablation for liver tumors. J Clin Gastroenterol 2010;44(5):374-380

34 Lee HS, Park SY, Kim SK, et al. Thrombocytopenia represents a risk for deterioration of liver function after radiofrequency ablation in patients with hepatocellular carcinoma. Clin Mol Hepatol 2012;18(3):302-308

35 Pang Q Qu K, Zhang JY, et al. The prognostic value of platelet count in patients with hepatocellular carcinoma: a systematic review and meta-analysis. Medicine (Baltimore) 2015;94(37):e1431 
36 De Pietri L, Bianchini M, Montalti R, et al. Thrombelastographyguided blood product use before invasive procedures in cirrhosis with severe coagulopathy: A randomized, controlled trial. Hepatology 2016;63(2):566-573

37 O'Leary JG, Greenberg CS, Patton HM, Caldwell SH. AGA clinical practice update: coagulation in cirrhosis. Gastroenterology 2019;157(1):34-43.e1

38 Lee LH, Hwang JI, Cheng YC, et al. Comparable outcomes of ultrasound versus computed tomography in the guidance of radiofrequency ablation for hepatocellular carcinoma. PLoS One 2017;12(1):e0169655

39 Kitada T, Murakami T, Kuzushita N, et al. Effectiveness of real-time virtual sonography-guided radiofrequency ablation treatment for patients with hepatocellular carcinomas. Hepatol Res 2008;38(6):565-571

40 Kim JE, Kim YS, Rhim H, et al. Outcomes of patients with hepatocellular carcinoma referred for percutaneous radiofrequency ablation at a tertiary center: analysis focused on the feasibility with the use of ultrasonography guidance. Eur J Radiol 2011;79(2):e80-e84

41 Clasen S, Boss A, Schmidt D, et al. MR-guided radiofrequency ablation in a 0.2-T open MR system: technical success and technique effectiveness in 100 liver tumors. J Magn Reson Imaging 2007;26(4):1043-1052

42 Clasen S, Rempp H, Boss A, et al. MR-guided radiofrequency ablation of hepatocellular carcinoma: long-term effectiveness. J Vasc Interv Radiol 2011;22(6):762-770

43 Clasen S, Rempp H, Hoffmann R, Graf H, Pereira PL, Claussen $\mathrm{CD}$. Image-guided radiofrequency ablation of hepatocellular carcinoma (HCC): is MR guidance more effective than CT guidance? Eur J Radiol 2014;83(1):111-116

44 Yuan C, Yuan Z, Cui X, et al. Efficacy of ultrasound-, computed tomography-, and magnetic resonance imaging-guided radiofrequency ablation for hepatocellular carcinoma. J Cancer Res Ther 2019;15(4):784-792

45 Lee MW, Rhim H, Cha DI, et al. Percutaneous radiofrequency ablation of hepatocellular carcinoma: fusion imaging guidance for management of lesions with poor conspicuity at conventional sonography. AJR Am J Roentgenol 2012;198(6):1438-1444

46 Song KD, Lee MW, Rhim H, et al. Percutaneous US/MRI fusionguided radiofrequency ablation for recurrent subcentimeter hepatocellular carcinoma: technical feasibility and therapeutic outcomes. Radiology 2018;288(3):878-886

47 Krücker J, Xu S, Glossop N, et al. Electromagnetic tracking for thermal ablation and biopsy guidance: clinical evaluation of spatial accuracy. J Vasc Interv Radiol 2007;18(9):1141-1150

48 Tomonari A, Tsuji K, Yamazaki H, et al. Feasibility of the virtual needle tracking system for percutaneous radiofrequency ablation of hepatocellular carcinoma. Hepatol Res 2013;43(12):1352-1355

49 Engstrand J, Toporek G, Harbut P, Jonas E, Nilsson H, Freedman J. Stereotactic CT-guided percutaneous microwave ablation of liver tumors with the use of high-frequency jet ventilation: an accuracy and procedural safety study. AJR Am J Roentgenol 2017;208(1):193-200

50 Odisio BC, Richter M, Aloia TA, et al. Use of prophylactic antibiotics to prevent abscess formation following hepatic ablation in patients with prior enterobiliary manipulation. J Gastrointest Surg 2016;20(8):1428-1434

51 Bhatia SS, Spector S, Echenique A, et al. Is antibiotic prophylaxis for percutaneous radiofrequency ablation (RFA) of primary liver tumors necessary? Results from a single-center experience. Cardiovasc Intervent Radiol 2015;38(4):922-928

52 Livraghi T, Solbiati L, Meloni MF, Gazelle GS, Halpern EF, Goldberg SN. Treatment of focal liver tumors with percutaneous radio-frequency ablation: complications encountered in a multicenter study. Radiology 2003;226(2):441-451

53 Hsieh YC, Limquiaco JL, Lin CC, Chen WT, Lin SM. Radiofrequency ablation following artificial ascites and pleural effusion creation may improve outcomes for hepatocellular carcinoma in high-risk locations. Abdom Radiol (NY) 2019;44(3): 1141-1151

54 Zhang M, Liang P, Cheng ZG, Yu XL, Han ZY, Yu J. Efficacy and safety of artificial ascites in assisting percutaneous microwave ablation of hepatic tumours adjacent to the gastrointestinal tract. Int J Hyperthermia 2014;30(2):134-141

55 Song I, Rhim H, Lim HK, Kim YS, Choi D. Percutaneous radiofrequency ablation of hepatocellular carcinoma abutting the diaphragm and gastrointestinal tracts with the use of artificial ascites: safety and technical efficacy in 143 patients. Eur Radiol 2009;19(11):2630-2640

56 Hermida M, Cassinotto C, Piron L, et al. Percutaneous thermal ablation of hepatocellular carcinomas located in the hepatic dome using artificial carbon dioxide pneumothorax: retrospective evaluation of safety and efficacy. Int J Hyperthermia 2018;35(1):90-96

57 Nishimura M, Nouso K, Kariyama K, et al. Safety and efficacy of radiofrequency ablation with artificial ascites for hepatocellular carcinoma. Acta Med Okayama 2012;66(3):279-284

58 ChaJ, Rhim H, LeeJY, etal. Percutaneous radiofrequency ablation of hepatocellular carcinoma: assessment of safety in patients with ascites. AJR Am J Roentgenol 2009;193(5):W424-9

59 Sherwani AK, Weis BA, Guynan J, et al. Evaluation of post-procedure hemorrhage in patients with ascites undergoing treatment of hepatic malignancy with thermal ablation utilizing microwave technology. J Clin Oncol 2018;34(15):e15582 